\title{
A Genetics Laboratory Module Involving Selection and Identification of Lysine Synthesis Mutants in the Yeast Saccharomyces cerevisiae
}

\author{
JILL B. KEENEY ${ }^{1 *}$ AND RUTH REED ${ }^{2}$ \\ Department of Biology ${ }^{1}$ and Department of Chemistry, ${ }^{2}$ Juniata College, Huntingdon, Pennsylvania 16652
}

We have developed a laboratory exercise, currently being used with college sophomores, which uses the yeast Saccharomyces cerevisiae to convey the concepts of amino acid biosynthesis, mutation, and gene complementation. In brief, selective medium is used to isolate yeast cells carrying a mutation in the lysine biosynthesis pathway. A spontaneous mutation in any one of three separate genetic loci will allow for growth on selective media; however, the frequency of mutations isolated from each locus differs. Following isolation of a mutated strain, students use complementation analysis to identify which gene contains the mutation. Since the yeast genome has been mapped and sequenced, students with access to the Internet can then research and develop hypotheses to explain the differences in frequencies of mutant genes obtained.

The yeast Saccharomyces cerevisiae is a commonly used organism in cell and molecular biology research (reviewed in reference 9). The simple growth requirements and rapid division time of yeast cells make them convenient for laboratory exercises in microbiology, cell biology, and genetics (4). The laboratory exercise described here uses the lysine synthesis pathway to explore the concepts of amino acid biosynthesis, mutation, and complementation analysis. Laboratory exercises involving yeast adenine mutants have previously been published $(5,10)$. The system we describe requires a more in-depth study of a biochemical pathway, as the selection is based on the use of a specific nitrogen source. Additionally, mutations at several genetic loci are selected so that complementation testing can be used to identify the mutant locus. Finally, the resulting mutation may be in either an enzyme or a regulatory protein, so that a full understanding of the exercise requires a student to comprehend how protein function relates to phenotype.

Laboratory strains of yeast, in the haploid state, are either a or a mating type, as determined by the MATa or MATa locus, respectively. A cross between haploids of different mating types forms a stable diploid. Wild-type (e.g., prototrophic) yeasts have no essential amino acids. Thus, yeasts are able to form colonies on minimal media containing a carbon source (usually glucose), a nitrogen source (usually ammonium sulfate), and a minimal medium base containing inorganic salts and vitamins.

Many of the yeast amino acid biosynthetic pathways have been studied in detail, and it has been found that some amino acids (or synthesis intermediates) can be utilized by the yeast cell as a nitrogen source. In yeast and higher fungi, lysine biosynthesis is achieved via the a-aminoadipate pathway (Fig. 1) $(1,11)$. The a-aminoadipate, a precursor in this pathway, can be used in place of ammonium sulfate as a nitrogen source by $S$. cerevisiae, but only in strains carrying a mutation in either the LYS2, LYS5, or LYS14 gene $(2,11)$. (Note that by

*Corresponding author. Mailing address: Department of Biology, Juniata College, 1700 Moore St., Huntingdon, PA 16652. Phone: (814) 641-3577. Fax: (814)641-3685. E-mail: keeney@juniata.edu. standard yeast genetic nomenclature, all genes are given a three-letter designation followed by a number. Wild-type genes are written in uppercase, e.g., LYS2. Mutant genes are written in lowercase and often referred to by a specific mutation number, e.g., lys2-801.) Thus, a-aminoadipate medium can be used to select for spontaneous or induced mutations in these genes, allowing for a mechanistically simple but cognitively informative laboratory exercise on mutation, selection, and complementation.

For the selection, a yeast strain is grown on rich medium and then replica plated onto medium containing aaminoadipate and lysine. Following incubation, mutant papillae are readily visible. Each colony arises from a cell that acquired a spontaneous mutation in lys2, lys5, or lys 14 . Mutant colonies are then single-colony purified by a second round of growth on selective media. Complementation analysis is used to determine which locus carries the mutation conferring a-aminoadipate resistance.

The biochemical basis for selection on a-aminoadipate can be explained in the context of the lysine biosynthesis pathway. The LYS2 and LYS5 genes each code for a separate subunit of the enzyme a-aminoadipate reductase, which con-

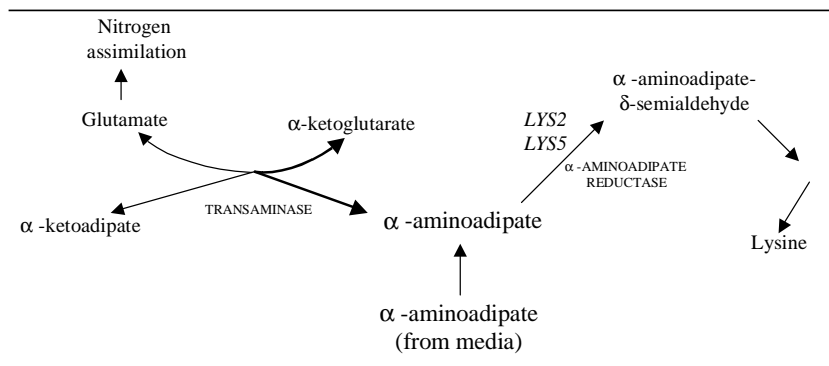

FIG. 1. An overview of the a-aminoadipate pathway for lysine biosynthesis. In a cell growing with abundant nitrogen, a-aminoadipate, produced from the transaminase reaction, is converted to lysine via several biochemical steps. Mutations in the lys 2 and lys 5 genes (encoding the aaminoreductase enzyme) result in sufficient levels of the reverse reaction to allow nitrogen assimilation using aaminoadipate as the nitrogen source. 
verts a-aminoadipate into a-aminoadipate-semialdehyde (8). The inactivation of a-aminoadipate reductase (via a mutation in LYS2 or LYS5) allows a-aminoadipate in the medium to serve as a nitrogen donor to a-ketoglutarate. This occurs by a reversal of the transamination step shown in Fig. 1. The glutamate formed in this reaction then serves as a nitrogen source for the synthesis of other amino acids. In a wild-type (LYS2 LYS5) strain, the active reductase enzyme readily converts a-aminoadipate to a-aminoadipate-semialdehyde, thus greatly reducing the reverse transaminase reaction (11).

LYS14 mutations are also selected on a-aminoadipate medium. The LYS14 gene encodes a DNA regulatory protein that increases the expression of several genes involved in lysine biosynthesis, including LYS 2 and LYS5 $(3,6)$. Thus, in the absence of functional LYS14 gene product, the level of a-aminoadipate reductase is sufficiently reduced for aaminoadipate to be the primary nitrogen source for the cell. Additionally, the lysine present in the selective medium represses activation by the LYS14 product.

Once a-aminoadipate-resistant mutants are isolated, the gene locus containing the mutation that confers resistance is identified by complementation analysis. "Tester" yeast strains containing a known mutation in lys2, lys 5 , or lys 14 are each mated with the a-aminoadipate-resistant mutant strains, thus forming stable diploids. The diploids are then replica plated onto medium lacking lysine. Growth of the diploid on this medium indicates a functional lysine synthesis pathway. Thus, the haploid parents contain mutations at different genetic loci, and the wild-type loci from each parent are able to "complement" each other, generating a functional pathway. The lack of diploid growth on medium lacking lysine indicates that the parent haploid strains harbor a mutation at the same genetic locus, and neither is able to provide a functional gene product to the synthesis pathway.

\section{MATERIALS*}

S. cerevisiae strains (readily available upon request to Jill Keeney):

F763 (MATa trp1 ura3 gal2)

Tester strains

4NN-214 (MATa lys2-801 ade2-10 ura3-52)

DG752 (MATa lys5)

8201-7A (MATa lys 14) (available as ATCC 204874)

Yeast-peptone-dextrose (YPD) plates

a-Aminoadipate plates containing lysine

Synthetic dextrose medium lacking lysine (SD-lysine)

Sterile toothpicks

Replica blocks and velvet squares

Incubator at $30^{\circ} \mathrm{C}$ (optional)

*Instructions for media preparation are given at the end of the paper.

\section{PROCEDURE}

Notes. (i) The actual hands-on time for this exercise is very short; we have the students do the replica plating and streaking at the beginning of a lab period, prior to the start of a longer laboratory exercise.

(ii) Throughout the protocol, incubation times are given for $30^{\circ} \mathrm{C}$. Incubation at room temperature will generally require 3 to 5 additional days. Storing the plates between lab periods can accommodate variations in laboratory schedules. Following any incubation step in the protocol, plates can be placed at $4^{\circ} \mathrm{C}$ until the next lab period. This will prevent overgrowth of cells.

Growth of yeast strains and isolation of mutants. Inoculate a plate of YPD agar with strain F763. This should be spread as a 3 by $5-\mathrm{cm}$ patch and allowed to grow for 1 to 3 days at $30^{\circ} \mathrm{C}$ or 4 to 5 days at room temperature. One patch will provide enough mutants for four students. At the same time, stock plates containing patches of the tester strains should also be prepared on YPD agar plates. Prepare one plate containing the three tester strains for every four students and incubate at room temperature until needed.

Using a replica block, transfer the F763 cells to a plate of a-aminoadipate medium (recipes are given at the end of this article). After approximately 1 week at $30^{\circ} \mathrm{C}$, colonies (called papillae) should be visible against a background of cells. These papillae result from the clonal growth of a mutant cell.

Colony purification of mutants. Divide a fresh aaminoadipate plate in half, and label the halves mutant 1 and mutant 2. Touch a sterile toothpick to one of the papillae, and streak the cells along the top third of the area designated for unknown 1 on the a-aminoadipate plate (Fig. 2, area A). Be sure to take care when streaking colonies with the sterile toothpicks, as the surface of the agar is fragile and easily gouged. Using a fresh toothpick, continue streaking the yeast from the end of area A into the middle third of your plate (Fig. 2, area B). With a third toothpick, spread the streak into the final portion of the plate (Fig. 2, area C). Understand that while the yeast cells will not be visible, the cells from the original colony are being spread out to the point where individual cells will now give rise to distinct colonies. Similarly, pick a second colony and streak it on the other half of

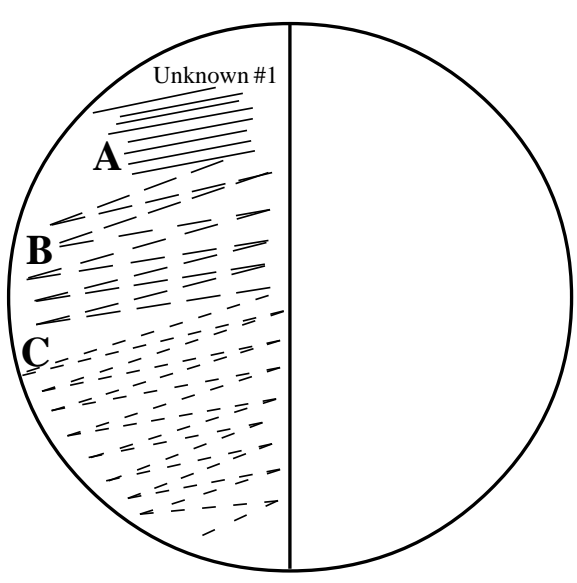

FIG. 2. Diagram of the procedure of streaking yeast cells to obtain single colonies. 
the plate in the area marked unknown 2. Incubate the plates at $30^{\circ} \mathrm{C}$ for 1 week until purified colonies appear in each sector.

Growth of mutants and tester strains for complementation test. Using sterile toothpicks, transfer a single colony arising from each of the unknowns onto a sterile YPD plate, making a separate thick vertical strip that runs the length of the plate for each mutant (Fig. 3). Four mutants can be inoculated on one YPD plate. Be sure that the cells from the colony are evenly spread across the plate. On a separate YPD plate, similarly inoculate a small amount of each of the three tester yeast strains (previously grown on YPD), making a single vertical strip for each tester strain. Incubate all plates at $30^{\circ} \mathrm{C}$ overnight.

Mating and complementation testing. By replica plating, transfer the strips from the plate of tester strains onto a sterile velvet square. Then, place the YPD plate containing the unknowns onto the same velvet so that the tester strains and the unknown mutants are oriented perpendicular to each other (Fig. 3A). Tap lightly on the plate to transfer the cells. Finally, transfer the resulting grid to a fresh YPD plate. The a-aminoadipate-resistant MATa cells and the MATa tester cells will form diploids at the intersections where they come into contact. Mating requires $4 \mathrm{~h}$ at $30^{\circ} \mathrm{C}$; alternatively, plates may be incubated overnight. Actively mating yeast cells produce mating pheromone, causing the cell to form a peculiar pear shape, often called a shmoo or propeller. This cell shape can be observed microscopically in actively mating cells (i.e., after $4 \mathrm{~h}$ at $30^{\circ} \mathrm{C}$ ).

By replica plating, transfer the cell grid from the YPD plate to an SD-lysine plate and incubate at $30^{\circ} \mathrm{C}$ overnight. Areas where two strains with complementing mutations have
A

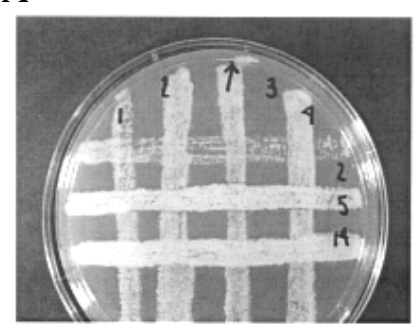

YPD

\section{B}

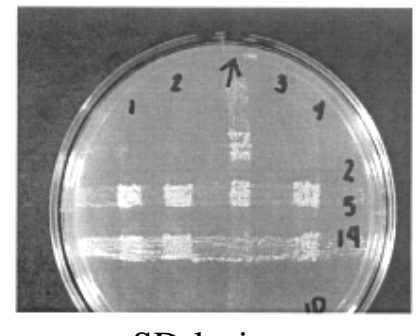

SD-lysine
FIG. 3. The complementation test. (A) Yeast strains containing mutations in lys2, lys5, and lys 14 (horizontal lines) were replicated onto YPD medium perpendicular to aaminoadipate-resistant yeast strains (vertical lines) of opposite mating type. After allowing mating to occur, the plate was replica plated onto medium lacking lysine. (B) The replica plate to medium lacking lysine, showing the results of the complementation test. Unknowns 1, 2, and 4 contain a mutation in lys 2 , as shown by the lack of growth at the intersection with the lys 2 strain. Unknown 3 contains a mutation in lys 14 . Note the leaky growth phenotype of the lys 14 strains, indicated by the light growth outside the squares of complementing growth. mated will show growth. The intersecting region of the unknown and the known producing no growth defines the mutation, as shown in Fig. 3B. Note that lys 14 mutations are leaky; the entire strip will show slow growth on SD-lysine. The mutated lys 14 gene product is unable to activate the lysine pathway, but a low level of basal promoter activity remains in the absence of this activator.

\section{EXPECTED RESULTS AND STUDENT OUTCOMES}

A large-scale screen for a-aminoadipate-resistant mutants has been conducted three times, and the results indicate that although similar results are obtained in separate screens, the strain background and variations in the medium preparation affect the percentage of each mutant isolated. In a research project involving isolation of 345 a-aminoadipate resistant mutants of strain F763, $334(97 \%)$ of the mutants were altered in lys $2,8(2 \%)$ were altered in lys 5 , and $3(1 \%)$ were altered in lys 14. Analysis of 115 F763 mutants isolated in our laboratory course yielded 94 lys 2 (82\%) mutations, 7 lys5 (6\%) mutations, and 14 lys 14 (12\%) mutations. Isolation of $\sim 200$ mutants from strain GRF167 (MATa his3 ura3) resulted in a majority of lys 2 mutants, with $4 \%$ lys 5 mutants and 0 lys 14 mutants. As this latter selection was done in a different semester than the previous one, it is unknown whether the absence of lys 14 mutants was due to the strain difference or differences in medium preparation. In tallying the results, we emphasize that this exercise relies on spontaneous mutations, which are then isolated through positive selection. Students are often amazed at the number of mutations that arise from a seemingly small number of cells, without application of a mutagen. Since a majority of our students are health professions majors, this provides an excellent background for a discussion of the genetic mechanism of the rapid increase in antibiotic-resistant strains of bacteria observed in medicine over the past decade.

At the conclusion of the exercise, students are required to write an individual report, 4 to 6 pages in length, which includes Introduction, Materials and Methods, Results, Discussion, and Literature Cited sections. The report must include an explanation of the biochemical basis of the aaminoadipate selection. For the discussion, students are expected to conduct a literature search and use the articles they find to explain their results. Basic goals are to have the students gain an understanding of how mutations in different genes in a biochemical synthesis pathway can give rise to similar phenotypes (in this case, a-aminoadipate resistance) and to understand how variations in phenotype relate to gene function. To this end, we give the students two basic questions to be answered in the discussion: (i) why is the frequency of lys 2 mutations obtained so much higher than that of the other mutations? and (ii) why is the lys 14 mutation leaky? If Internet access is available, students can be directed to the Saccharomyces genome website (7). A search by gene name will bring up the general gene information, the gene sequence, and links to references about the gene. Students may notice that LYS2 (4,179 bases) is much larger than 
the LYS5 gene ( 819 bases) and thus is simply a bigger target for genetic mutation. Students may also theorize how the function and nature of the protein affect what kind of mutations will abolish protein function. For example, the LYS2 structural gene may be much more sensitive to mutational changes than LYS5 or LYS14. Perhaps this subunit contains the active site or a critical structural domain. Students often come up with theories proposing hot spots for mutation, which may also be true. The LYS14 gene product activates several different genes and thus by nature is likely to have a less rigid structure that is tolerant of mutations. We encourage the students to research and think about their own theories to explain the results. Beyond answering the questions listed above, the students are encouraged to discuss relevant information they find concerning the pathway. We expect students to find references relating to the biochemical function of a-aminoadipate reductase and the regulatory function of lys 14 and to be able to summarize the information in the context of this experiment. Additionally, some students discuss other genes in the pathway and/or the medical significance of lysine.

\section{EXTENSIONS}

Close observation of the SD-lysine plates may demonstrate two other genetic phenomena. Some of the selected mutants will revert to lysine prototrophy at a high frequency, as indicated by papillae that appear outside the intersecting regions on the SD-lysine plate. Additionally, intragenic recombination may occur in the diploids. This can be seen as papillae growing at the junctions of two noncomplementing strains.

Although we have not tried it, this assay could presumably be used to test mutagenic sources by observing the number of papillae generated on uniform-sized patches exposed to UV light or other mutagens. It is expected that an unexposed control patch would have fewer papillae than a patch that has been exposed to a mutagen.

In summary, this laboratory exercise can be used to teach the concepts of genetic selection of spontaneous mutations in the context of a biochemical pathways and complementation analysis. At the conclusion of this exercise, we expect the students to understand the following:

- how a cell can alter enzyme kinetics within a biochemical pathway to utilize an alternate nitrogen source,

- that the spontaneous mutation rate within a cell will generate a significant number of mutations that can be selected for growth under specific conditions,

- how gene function relates to phenotype, and

- genetic complementation.

Most students enjoy this lab and feel quite a sense of accomplishment after completing the written report. Finally, it is worth noting that several exceptionally bright and motivated juniors and seniors currently doing independent research have commented that their desire to do research was inspired by the "lys lab." They found that the investigative nature of the lab in relation to gene function inspired them to pursue fur- ther genetic research as undergraduates.

\section{MEDIA PREPARATION}

A class of 15 students will need $\sim 20$ a-aminoadipate plates, $\sim 45$ YPD plates, and $\sim 15$ SD-lysine plates. The recipes given below will each yield $\sim 20$ plates, at a final concentration of $2 \%$ agar. The agar and each medium recipe is for $250 \mathrm{ml}$ of a $2 \mathrm{x}$-concentrated stock. We make up the agar and medium stock solutions in 500-ml bottles, autoclave them, and store them at room temperature. The agar can then be melted in a microwave oven and mixed with any $2 x$ medium solution to yield a $1 \mathrm{x}$ medium of choice ready for pouring. Always add molten agar to the liquid medium; it mixes better.

4\% Agar.

$250 \mathrm{ml}$ of $2 \mathrm{x}$ agar

$10 \mathrm{~g}$ of agar

$250 \mathrm{ml}$ of water

Autoclave for $15 \mathrm{~min}$ at $121^{\circ} \mathrm{C}$ in a $500-\mathrm{ml}$ bottle.

YPD plates.

$250 \mathrm{ml}$ of $2 \mathrm{x}$ YPD (makes $\sim 20$ plates)

$5 \mathrm{~g}$ of Bacto yeast extract

$10 \mathrm{~g}$ of Bacto peptone

$0.08 \mathrm{~g}$ of tryptophan

$10 \mathrm{~g}$ of dextrose

$250 \mathrm{ml}$ of $\mathrm{H}_{2} \mathrm{O}$

Dissolve yeast extract, peptone, tryptophan, and dextrose in the $\mathrm{H}_{2} \mathrm{O}$. Place the ingredients in a $500-\mathrm{ml}$ bottle and autoclave for $15 \mathrm{~min}$ at $121^{\circ} \mathrm{C}$. Add $250 \mathrm{ml}$ of $4 \%$ agar (molten, sterile), swirl gently to mix well, and pour plates.

a-Aminoadipate plates (with lysine, tryptophan, and uracil).

$250 \mathrm{ml}$ of $2 \mathrm{x}$ a-aminoadipate medium (makes $\sim 20$ plates)

$1 \mathrm{~g}$ of yeast nitrogen base (without amino acids and

without nitrogen)

$15 \mathrm{mg}$ of lysine

$11.2 \mathrm{mg}$ of uracil

$80 \mathrm{mg}$ of tryptophan

$10 \mathrm{~g}$ of dextrose

$250 \mathrm{ml}$ of $\mathrm{H}_{2} \mathrm{O}$

Dissolve nitrogen base, lysine, uracil, tryptophan, and dextrose in the $\mathrm{H}_{2} \mathrm{O}$; autoclave for $15 \mathrm{~min}$ at $121^{\circ} \mathrm{C}$ in a $500-\mathrm{ml}$ bottle. After autoclaving, add $25 \mathrm{ml}$ of a-aminoadipate solution (below) and swirl to mix well. Add $250 \mathrm{ml}$ of $4 \%$ agar (molten, sterile). Swirl gently to mix well, and pour plates.

Preparation of a-aminoadipate solution.

$2 \mathrm{~g}$ of DLa-aminoadipate (Sigma A0637, keep desiccated, refrigerated)

$30 \mathrm{ml}$ of $\mathrm{H}_{2} \mathrm{O}$

With $1 \mathrm{M} \mathrm{KOH}$, bring to $\mathrm{pH} 6$ to dissolve (+/- $12 \mathrm{ml})$. Filter sterilize.

SD-lysine plates (synthetic medium, no lysine, with tryptophan, adenine, and uracil included to cover other auxotrophic markers).

$250 \mathrm{ml}$ of $2 \mathrm{x}$ SD-lysine medium (makes $\sim 20$ plates)

$0.85 \mathrm{~g}$ of yeast nitrogen base (without amino acids and 
without ammonium sulfate)

$2.5 \mathrm{~g}$ of ammonium sulfate

$11.2 \mathrm{mg}$ of uracil

$92.5 \mathrm{mg}$ of adenine

$80 \mathrm{mg}$ of tryptophan

$10 \mathrm{~g}$ of dextrose

$250 \mathrm{ml}$ of $\mathrm{H}_{2} \mathrm{O}$

Dissolve nitrogen base, ammonium sulfate, uracil, adenine, tryptophan, and dextrose in the $\mathrm{H}_{2} \mathrm{O}$; autoclave for $15 \mathrm{~min}$ at $121^{\circ} \mathrm{C}$ in a $500-\mathrm{ml}$ bottle. Swirl to mix well. Add $250 \mathrm{ml}$ of $4 \%$ agar (molten, sterile). Swirl gently to mix well, and pour plates.

Ordering information. Sigma Chemical Company supplies adenine sulfate (A9126), tryptophan (T0254), uracil (U0750), and DLa-aminoadipate (A0637). Yeast nitrogen base, ammonium sulfate, dextrose, and agar can be purchased from any supplier of standard medium reagents.

\section{ACKNOWLEDGMENTS}

Many thanks to former Juniata College students Barry Ide and Greta Schrift for their help with making media, testing the system, and assisting in the classroom.

\section{REFERENCES}

1. Bhattacharjee, J. K. 1985. a-Aminoadipate pathway for the biosynthesis of lysine in lower eukaryotes. Crit. Rev. Microbiol. 12:131-151.
2. Chattoo, B. B., F. Sherman, D. A. Azubalis, T. A. Fjellstedt, D. Mehnert, and M. Ogur. 1979. Selection of lys 2 mutants of the yeast Saccharomyces cerevisiae by the utilization of a-aminoadipate. Genetics 93:51-65.

3. Feller, A., E. Dubois, F. Ramos, and A. Pierard. 1994. Repression of the genes for lysine biosynthesis in Saccharomyces cerevisiae is caused by limitation of Lys 14-dependent transcriptional activation. Mol. Cell. Biol. 14:6411-6418.

4. Manney, T. R., and M. L. Manney. 1992. Yeast: a research organism for teaching genetics. Am. Biol. Teacher 54:426.

5. Montelone, B. A., B. Williamson, and L. Mayo. 1995. Rapid transformation of a color mutant of yeast. Am. Biol. Teacher 57:171. 6. Ramos, F., E. Dubois, and A. Pierard. 1988. Control of enzyme synthesis in the lysine biosynthetic pathway of Saccharomyces cerevisiae. Evidence for a regulatory role of gene LYS14. Eur. J. Biochem. 171:171-176.

7. Stanford University. 25 August 1999. Saccharomyces Genome Database.

8. Storts, D. R., and J. K. Bhattacharjee. 1989. Properties of revertants of lys 2 and lys 5 mutants as well as alpha-aminoadipatesemialdehyde dehydrogenase from Saccharomyces cerevisiae. Biochem. Biophys. Res. Commun. 161:182-186.

9. Watson, J. D., N. H. Hopkins, J. W. Roberts, J. A. Steitz, and A. M. Weimer (ed.). 1987. Molecular biology of the gene, 4th ed., p. 550-594. Benjamin/Cummings, Menlo Park, Calif.

10. White, B. T. 1999. The red \& white yeast lab. Am. Biol. Teacher 61:600-604.

11. Zaret, K. S., and F. Sherman. 1985. a-Aminoadipate as a primary nitrogen source for Saccharomyces cerevisiae mutants. J. Bacteriol. 162:579-583.

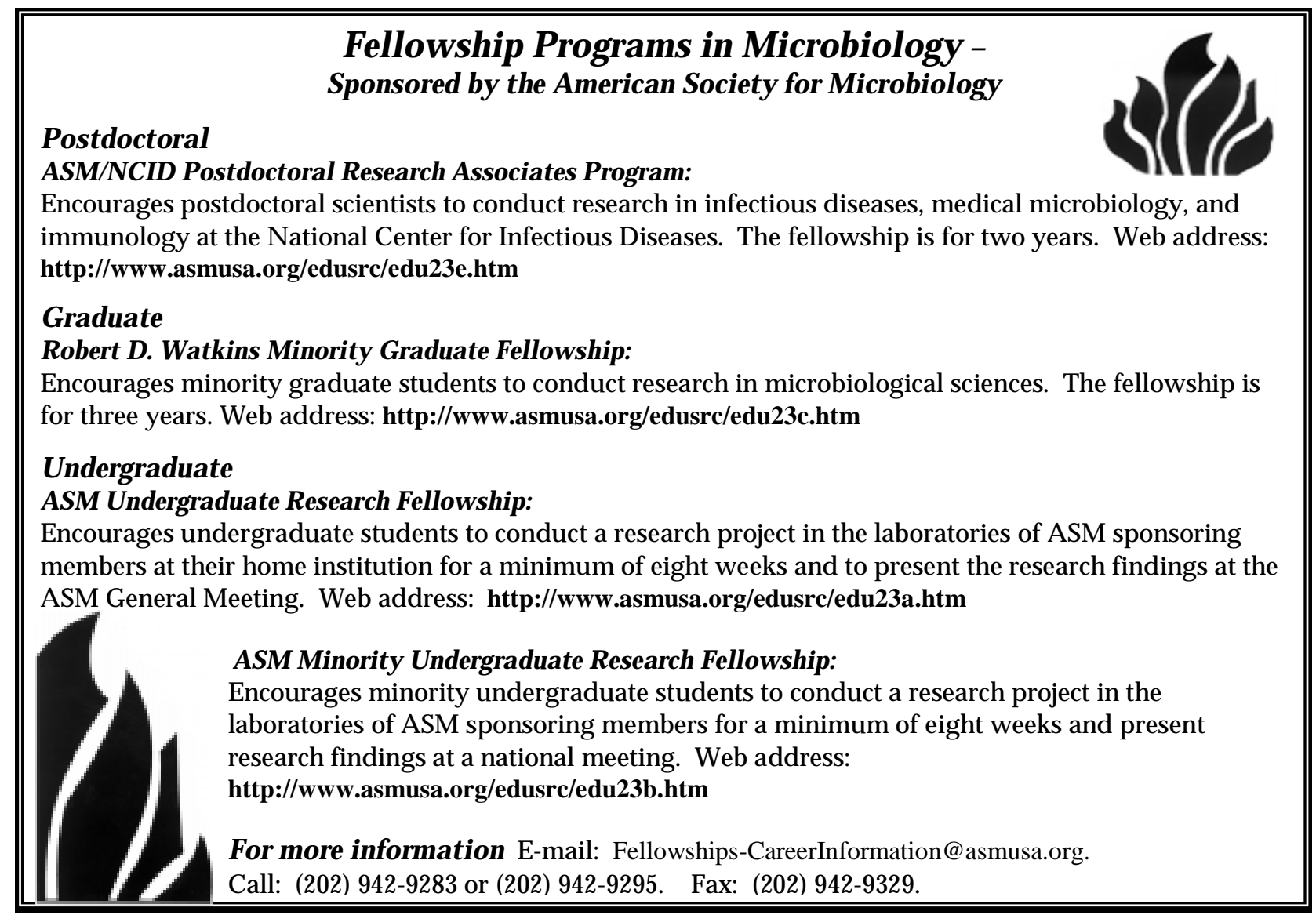

